\title{
Exploring the Use of Two Antennas for Crosscorrelation Spectrum Sensing
}

\author{
M.S. Oude Alink, A.R. Smeenge, A.B.J. Kokkeler, E.A.M. Klumperink, G.J.M. Smit and B. Nauta \\ Integrated Circuit Design / Computer Architecture for Embedded Systems \\ CTIT Research Institute, University of Twente, Enschede, The Netherlands \\ Email: m.s.oudealink@utwente.nl
}

\begin{abstract}
Spectrum sensing is one of the key characteristics of a cognitive radio. Energy detection provides maximum flexibility by not relying on any prior knowledge, but suffers from an SNRwall due to noise uncertainty. Crosscorrelation of the outputs of two receiver paths is a technique to reduce the noise level of the total receiver, and hence improves the SNR. The reduction of the noise is limited by correlated noise originating from shared components near the antenna. In this paper we explore the use of a separate antenna for each receiver for crosscorrelation spectrum sensing. One immediate advantage is that due to the removal of the splitter, which was necessary to interface the single antenna to two receivers, the SNR improves, significantly reducing the required measurement time. A lot of the noise correlation can be removed, leading to a lower residual noise floor. The noise at each antenna will still be partially correlated due to mutual coupling, spatial noise correlation and man-made noise. We show that some signal power can be lost in the sensing process due to partial decorrelation of the signal at the two antennas. Fortunately, this seems to be a problem only in highly mobile environments, which makes the use of two-antenna crosscorrelation spectrum sensing an interesting solution towards more reliable energy detection.
\end{abstract}

Index Terms-antennas, cognitive radio, crosscorrelation, energy detection, noise correlation, noise uncertainty, radiometer, SNR-wall, spectrum sensing

\section{INTRODUCTION}

C OGNITIVE RADIO (CR) is a paradigm to a more efficient usage of spectrum. Instead of being assigned to fixed frequency bands, a CR scans the spectrum and opportunistically uses available frequencies. Energy detection would be preferable, because it allows a CR to operate in any possible frequency band, without the requirement to know anything about the type of signals present in such a band. It has the disadvantage that due to uncertainty in the noise level, a minimum signal-to-noise ratio (SNR) exists below which a signal cannot be reliably detected [1]. This minimum SNR is called the SNR-wall.

Crosscorrelation has been proposed to reduce this SNRwall for energy detection [2], where a spectrum analyzer (SA) (which we will refer to as the crosscorrelation spectrum analyzer (XCSA)) uses crosscorrelation to lower the noise level. The idea is to have two receiver paths, such that the noise added by each path is uncorrelated with the noise in the other path. By crosscorrelating the output of two receiver paths, the uncorrelated noise is removed, which in [2] reduces the noise floor from $-150 \mathrm{dBm} / \mathrm{Hz}$ to $-170 \mathrm{dBm} / \mathrm{Hz}$. Intuitively, when the noise is eventually removed, any uncertainty of its power does not matter. Noise uncertainty can be minimized by on-line calibration, but there will always be some remaining uncertainty [3]. Therefore, the goal is to minimize the noise figure (NF) (higher SNR) and the noise correlation (higher SNR / less uncertainty).

The XCSA uses a single antenna followed by a passive splitter and resistive attenuators; most of the noise correlation originates from these components as they are shared by the two receivers. In this paper, we propose to use a separate antenna for each receiver to improve the NF ([2], [4]) and to reduce the noise correlation by removing the splitter (and hence the direct connection between the two receivers). The use of multiple antennas is getting more and more accepted, as it is used in diversity receivers, for beamforming and MIMO-systems, and has been proposed in many other works on spectrum sensing. Although this seems a straightforward next step, we will show that the consequences for the noise and signal powers in the crosscorrelation spectrum sensing process are nontrivial.

This paper is organized as follows. In section II we will briefly summarize the key aspects of crosscorrelation spectrum sensing and the prototype of [2]. We will then start to investigate the impact of using two antennas on the noise level in section III, which includes some measurements. A discussion on the impact on signal detection is given in section IV, while we end with conclusions in section V.

\section{Crosscorrelation Spectrum Sensing}

For the purpose of noise analysis, the receiver chain, which typically includes an LNA, mixer, amplifiers, prefilters and an analog-to-digital converter (ADC), is modeled as a linear device that only adds some noise. For a crosscorrelation system (part of) the receiver chain is duplicated, where uncorrelated noise is added in each of the two paths, see Fig. 1. When these separate paths generate correlated noise, e.g. through shared components, such as the splitter resistors, or (parasitic) coupling, such as the antenna(s) or the power supply, the correlated noise part can be moved to $n_{\text {corr }}$ without loss of generality. The signal plus correlated noise $x$ is filtered in each path by a filter with bandwidth $W$ and then sampled at the Nyquist rate, resulting in the complex receiver outputs $r_{1}$ and $r_{2}$. The energy detection is performed by multiplying $r_{1}$ and $\overline{r_{2}}\left(\overline{r_{2}}\right.$ denotes the complex conjugate of $\left.r_{2}\right)$, and averaging the result. This final output $y$ is used to decide whether a signal is present or not. 


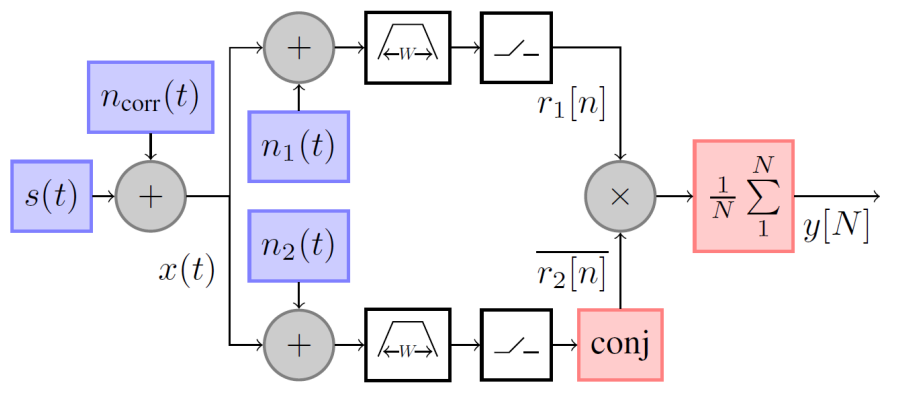

Fig. 1. Energy detection using crosscorrelation.

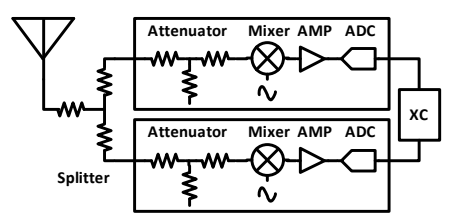

(a) Prototype in [2]

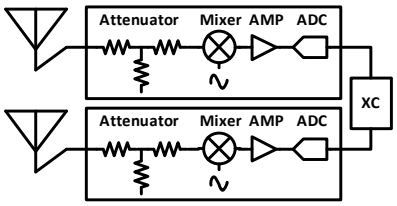

(b) Two-antenna proposal
Fig. 2. Prototype (one antennas) and proposed system (two antennas).

Assuming the signal and all noise sources are uncorrelated, we find (with $\mathbb{E}[\cdot]$ denoting the expectation operator)

$$
\begin{aligned}
\mathbb{E}[y] & =\mathbb{E}\left[r_{1} \overline{r_{2}}\right]=\mathbb{E}\left[\left(x+n_{1}\right) \overline{\left(x+n_{2}\right)}\right] \\
& =\mathbb{E}\left[|x|^{2}\right]+\mathbb{E}\left[x \overline{n_{2}}\right]+\mathbb{E}\left[n_{1} \bar{x}\right]+\mathbb{E}\left[n_{1} \overline{n_{2}}\right] \\
& =\mathbb{E}\left[|x|^{2}\right]=\mathbb{E}\left[|s|^{2}\right]+\mathbb{E}\left[\left|n_{\text {corr }}\right|^{2}\right]=P_{s}+P_{n_{\text {corr }}}
\end{aligned}
$$

In other words, the uncorrelated noise power is removed using the crosscorrelation process, while the signal power and the correlated noise power remain. Note that for autocorrelation, $r_{1}=r_{2}$ and $n_{1}=n_{2}=0$, with $n_{\text {corr }}$ containing all of the noise of the receiver, which is not removed. The removal of the uncorrelated noise power increases the SNR, and therefore allows smaller signals to be detected.

The prototype presented in [2] uses one antenna, and then splits the signal into two paths using resistors, where each path is attached to its own receiver, consisting of a resistive attenuator, a mixer, amplifiers and an ADC. This is schematically depicted in Fig. 2a. The noise voltage generated by each resistor in the splitter and the attenuators will be present at the input of both receivers (although possibly with different amplitudes due to the network transfer) because there is no isolation between them. From measurements in [2], $P_{n_{\text {corr }}} \approx-170 \mathrm{dBm} / \mathrm{Hz}$, which leaves room for improvement.

Therefore, we propose the use of a separate antenna for each receiver, such as depicted in Fig. 2b. This scheme has the direct advantage of reducing the NF of the system (before crosscorrelation) by $6 \mathrm{~dB}$ due to the removal of the resistive splitter, reducing measurement time significantly. It is, however, not trivial what the noise correlation and the signal correlation on the two antennas will be. This is the topic of the next sections.

\section{Noise CORRElation In Two ANTEnNAS}

We will first briefly explain the origin of noise in a single antenna, and then proceed to describe factors introducing noise correlation in two antennas. We end this section with some measurements on noise correlation.

\section{A. Noise in an Antenna}

Black-body radiation is the electro-magnetic (EM)-radiation emitted by a black body at a given temperature. Real-life objects are not perfect black bodies (as can be observed in the visible range of the spectrum), but the approximation is very good in the radio frequency range [5]. From the RayleighJeans approximation (accurate for frequencies up to tens of $\mathrm{GHz}$ ), the radiation of a black body is

$$
B_{\text {black-body }} \approx \frac{2 k T}{\lambda^{2}}\left[\mathrm{~W} \cdot \mathrm{Hz}^{-1} \cdot \mathrm{m}^{-2} \cdot \mathrm{sr}^{-1}\right]
$$

with $k$ Boltzmann's constant $\left(1.38 \cdot 10^{-23} \mathrm{~J} / \mathrm{K}\right), T$ the absolute temperature in Kelvin, and $\lambda=c / f$ the wavelength, with $c$ the speed of light $\left(3.00 \cdot 10^{8} \mathrm{~m} / \mathrm{s}\right)$ and $f$ the frequency in $\mathrm{Hz}$.

The purpose of an antenna is to guide the energy of the incident EM field to the load. The maximum power that can be delivered by the antenna to the load is called the available power $P_{\mathrm{av}}$, with $S_{\mathrm{av}}$ the available power per Hertz. This maximum power transfer is obtained under conjugate matching, which is assumed from here on. The effective area $A_{e}$ of an antenna is defined as "the ratio of the available power at the terminals of a receiving antenna to the power flux density of a plane wave incident on the antenna from that direction, the wave being polarization-matched to the antenna" [6], and in general depends on direction, with $A_{e}(\theta, \phi)=$ $\frac{\lambda^{2}}{4 \pi} G(\phi, \theta) \quad\left[\mathrm{m}^{2}\right]$, and $G(\phi, \theta)$ the directional antenna gain. Therefore, assuming the antenna is completely surrounded by black bodies in thermal equilibrium

$$
\begin{aligned}
S_{\mathrm{av}} & =\int_{0}^{2 \pi} \int_{0}^{\pi} B_{\text {black-body }} A_{e}(\theta, \phi) \sin \theta \mathrm{d} \theta \mathrm{d} \phi \\
& =\frac{1}{2} \frac{2 k T}{\lambda^{2}} \frac{\lambda^{2}}{4 \pi} 4 \pi=k T[\mathrm{~W} / \mathrm{Hz}]
\end{aligned}
$$

where the factor $\frac{1}{2}$ in the second step is due to the unpolarized nature of the black-body radiation and the polarized reception of the antenna. The noise floor, taken as $k T$, with $T=290 \mathrm{~K}$, is then $-174 \mathrm{dBm} / \mathrm{Hz}$. So, even when the antenna itself is noiseless, which we assume from here on, it will deliver noise power to the attached receiver.

\section{B. Factors Introducing Noise Correlation in Two Antennas}

Many papers proposing multiple antenna spectrum sensing assume independent noise samples at each antenna, but several factors introduce correlated noise between two antennas.

1) Mutual Coupling: The EM-field impinging on one antenna induces a time-varying current in the antenna, which by itself generates an EM-field that may be received by the other antenna. This gives rise to mutual coupling. Mutual coupling will also introduce correlation of the noise generated by the receiver, as some noise generated in one receiver will be transmitted by its antenna, and then received by the other [7]. Hence, mutual coupling introduces correlated noise. 
2) Spatial Correlation: In [8], it is shown that, even when there is no antenna coupling, the thermal noise of two antennas can be correlated due to spatial correlation. The crosscorrelation function (ccf) $\gamma_{x, y}(\tau)=\mathbb{E}\left[V_{x}(t) V_{y}(t+\tau)\right]$ between the voltages induced on the antenna terminals by these noise sources depends on the distance $d$ between the antennas, the antenna patterns, and the polarization the antennas are sensitive to [8].

3) Man-made Noise: A receiver tries to receive information from one 'wanted' radiator, but there are many other radiators in that band, such as other transmitters, lossy cables, but also stellar objects and lightning. Other than the natural sources, these are often collectively combined into 'man-made noise'. Man-made noise, just like the black-body radiation, originates from many sources and arrives from many directions, so it will also have spatial correlation.

\section{Measurements}

To check whether noise is decorrelated in practice, we have performed some measurements. Two signal generators are used to represent two independent bandlimited white real noise sources, with a bandwidth of roughly $1 \mathrm{MHz}$. We can obtain the estimated power spectral density (PSD) $\mathcal{S}_{n}(f)$ for two noise sources $n_{\mathrm{src} 1}$ and $n_{\mathrm{src} 2}$ as:

$$
\mathcal{S}_{n}(f)=S_{n_{\text {src }}}(f) e^{-j 2 \pi f \tau_{1}}+S_{n_{\text {src } 2}}(f) e^{-j 2 \pi f \tau_{2}}
$$

where $\tau_{i}$ denotes the delay between the two antennas as seen from source $i$. By integrating over the bandwidth of interest $f_{b}$, we obtain the estimated power $\mathcal{P}_{n}$. By dividing $\mathcal{P}_{n}$ by the total power $P_{n}$, we obtain the noise correlation factor $\rho$ :

$$
\begin{aligned}
\rho \triangleq \frac{\mathcal{P}_{n}}{P_{n}} & =\frac{1}{f_{b}\left(P_{n_{\mathrm{sc} 1}}+P_{n_{\mathrm{sc} 2}}\right)} \int_{f_{0}-f_{b} / 2}^{f_{0}+f_{b} / 2} \mathcal{S}_{n}(f) \mathrm{d} f \\
& \approx \frac{1}{2} \int_{f_{0}-f_{b} / 2}^{f_{0}+f_{b} / 2} e^{-j 2 \pi f \tau_{1}}+e^{-j 2 \pi f \tau_{2}} \mathrm{~d} f
\end{aligned}
$$

In the second step it is assumed that $P_{n_{\mathrm{src} 1}} \approx P_{n_{\mathrm{src} 2}}$, as is the case for the measurements.

The setup resembles an XCSA as it would be used in practice, as shown in Fig. 3a, and uses two transmit and two receive antennas, positioned as shown in Fig. 3b. The receiver downconverts, amplifies and filters the signal before it is digitized. The crosscorrelation is then performed as described in [2]. By transmitting enough power, we make sure that the two noise sources dominate all man-made noise and interference.

Fig. 3c shows the theoretical correlation factor given by (5) and the measured correlation factor for an antenna separation of $50 \mathrm{~cm}: \tau_{1}=-\tau_{2}=\frac{d}{c} \approx 1.67 \mathrm{~ns}$. Clearly, the measurements do not follow (5). The measurements were repeated for other antenna separations, again showing similar chaotic results. We believe that the difference between (5) and measurements is caused by multi-path effects, which can be completely different for different frequencies. This would explain the erratic behavior of the correlation factor over frequency, and suggests that in a realistic (indoor) environment,

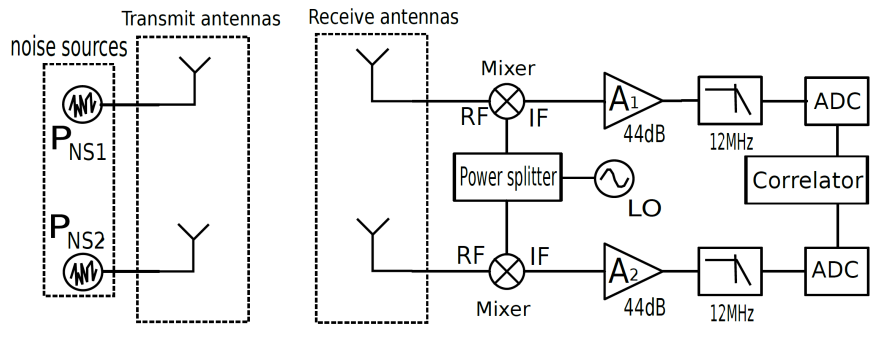

(a) Experimental setup

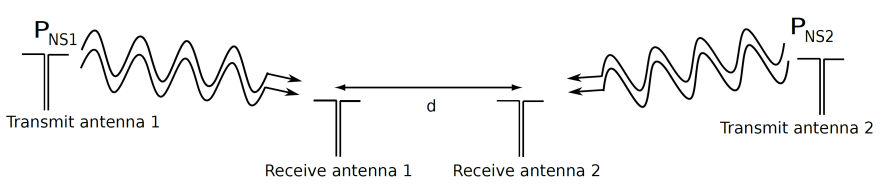

(b) Antenna placement

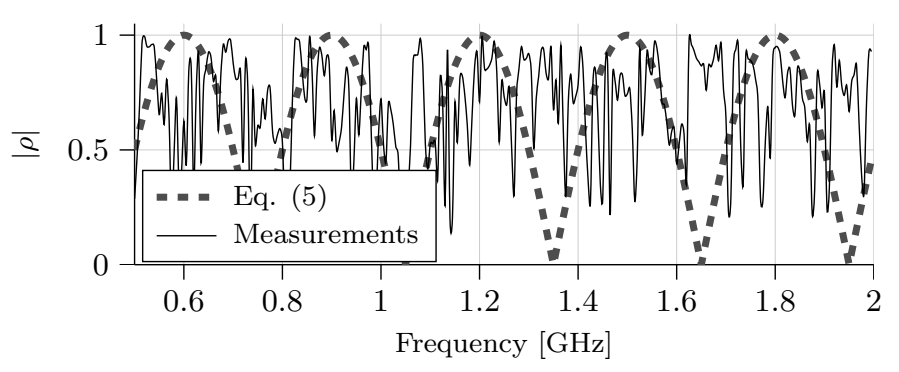

(c) Measurement results

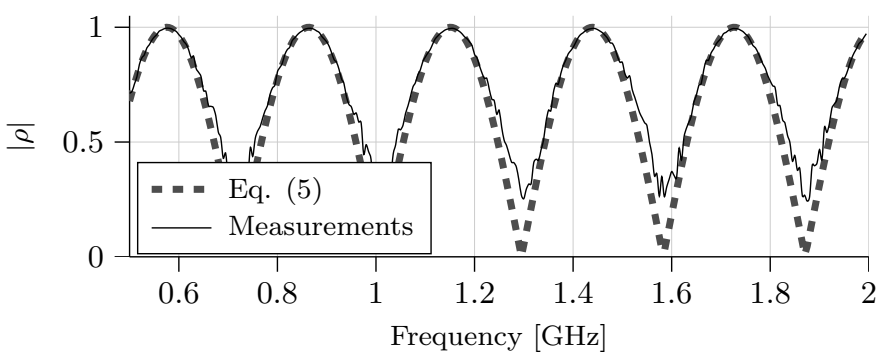

(d) Measurement results when using cables

Fig. 3. Measurement of the correlation factor for two noise sources.

such as the lab in which the experiments were conducted, the noise correlation can deviate a lot from a simple first-order model. Since we do not have access to an anechoic chamber to verify this, we repeated the experiments with cables of different lengths to replace the antennas and avoid multipath propagation, but still mimic the time delay. The results are shown in Fig. 3d, which clearly gives a much better match.

By looking at Fig. 3c and averaging $|\rho|,|\rho| \approx 0.7$. This means that with two antennas, the noise between the antennas is partially decorrelated.

\section{Signal Detection with Two Antennas}

When both the noise and the signal correlation are reduced by the same factor, the SNR is not changed, and neither will the ultimate sensitivity of a XCSA. Therefore, it is equally important to consider the signal correlation in the two-antenna XCSA. To achieve insight into this effect, we will explore the signal correlation for a few different scenarios assuming identically polarized antennas without mutual coupling. 


\section{A. Time-delay in line-of-sight (LOS)-connections}

In LOS-connections, there will be a time delay between the signal arriving on the first and the second antenna, depending on the orientation of the antennas with respect to the signal source. This time delay is unknown, because the angle of arrival of the signal is unknown. Mathematically, we find at the output of the crosscorrelator $\mathcal{S}_{s}(f)=S_{s}(f) e^{-j 2 \pi f \tau_{0}}$, where $\tau_{0}$ is the time delay. Hence, if we use the absolute value of $\mathcal{S}_{s}(f)$ (the PSD of a signal must be real and nonnegative), we find $\left|\mathcal{S}_{s}(f)\right|=S_{s}(f)$. Theoretically, the total power $\mathcal{P}_{s}$ in a band ranging from $f_{0}-\frac{f_{b}}{2}$ to $f_{0}+\frac{f_{b}}{2}$ is then found by integrating $\left|\mathcal{S}_{s}(f)\right|$ over this frequency range. However, the minimum bandwidth that can be estimated is inversely proportional to the measurement time, so we have to consider detection in a finite bandwidth $f_{b}$.

Each frequency within $f_{b}$ will experience a different phase shift between the two antennas. As, due to the time delay, the estimated spectrum can be complex, we again take the absolute value. For simplicity, we assume the signal is a white noise source in the band of interest, which is a good approximation of for example OFDM-signals. The estimated power then becomes

$$
\mathcal{P}_{s}=\left|\int_{f_{0}-\frac{f_{b}}{2}}^{f_{0}+\frac{f_{b}}{2}} \mathcal{S}_{s}(f) \mathrm{d} f\right|=P_{s}\left|\left(\frac{\sin \pi f_{b} \tau_{0}}{\pi f_{b} \tau_{0}}\right)\right|
$$

Clearly, for $f_{b}=0$ and/or $\tau_{0}=0, \mathcal{P}_{s}=P_{s}$. For larger $\tau_{0}$ and/or larger $f_{b}$, the measured power $\mathcal{P}_{s}$ becomes lower. If we maximally allow $1 \mathrm{~dB}$ loss of signal power, we find

$$
f_{b} \cdot \tau_{0} \lesssim 0.36
$$

Consider the use of the TV white space, with channels of $6 \mathrm{MHz}$. From (7) we find $\tau_{0} \leq 60 \mathrm{~ns}$. In a handheld device, the maximum distance $d_{\max }$ between the two antennas will be about $15 \mathrm{~cm}$, so the maximum time delay is $\tau_{\max }=\frac{d}{c} \approx$ $0.5 \mathrm{~ns}$, which gives negligible loss in signal power. Even when the antennas would be $10 \mathrm{~m}$ apart, the detected signal power will only be lowered by $0.3 \mathrm{~dB}$. So, in static LOSconnections, using two antennas for crosscorrelation spectrum sensing makes perfect sense.

\section{B. Movement in LOS-connections}

In the previous section, it was assumed that everything was static. Especially in a mobile context, the transmitter and/or the spectrum sensing device can move during the sensing process, which makes some parameters dynamic.

Consider the situation depicted in Fig. 4, where the transmitter moves during the sensing process, such that at time instant $t$, it is located at $p_{t}$, while the two receive antennas, located at $p_{x}$ and $p_{y}$, are stationary. The two receive antennas are separated by a distance $d_{a}$, and the transmitter moves at a uniform velocity $v$. We assume the signal power received by each antenna does not change, but the relative phase between the two antennas does (which can be considered a manifestation of the Doppler-effect). The largest change in phase is obtained if the transmitter moves as indicated: parallel

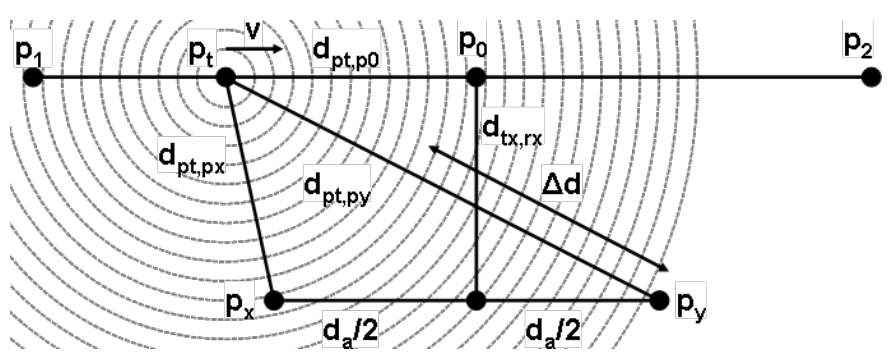

Fig. 4. Simple model of a moving transmitter and stationary two-antenna XCSA.

to the baseline of the antennas, passing point $p_{0}$ (at distance $d_{t x, r x}$ from the baseline) halfway during the sensing process, which takes $T$ seconds. The distance $d_{p_{1}, p_{2}}$ between $p_{1}$ and $p_{2}$ covered by the transmitter during the sensing process is thus $d_{p_{1}, p_{2}}=v T$.

We define $d_{p_{t}, p_{0}}(t)=v\left(\frac{T}{2}-t\right)$ and $\Delta d(t)=d_{p_{t}, p_{x}}(t)-$ $d_{p_{t}, p_{y}}(t)$, where $d_{p_{t}, p_{x}}(t) \geq 0$ and $d_{p_{t}, p_{y}}(t) \geq 0$. Then we find for the phase difference between the two receivers $\Delta \phi(t)=$ $2 \pi f \frac{\Delta d(t)}{c}$ (note that $\Delta d(t)$ can become negative). Considering a single frequency, the estimated PSD $\mathcal{S}_{s}(f)$ of the true PSD $S_{s}(f)$, after measuring for $T$ seconds, is then

$\mathcal{S}_{s}(f, T)=\frac{1}{T} \int_{0}^{T} S_{s}(f) e^{j \Delta \phi(t)} \mathrm{d} t=S_{s}(f) \cdot \frac{1}{T} \int_{0}^{T} e^{j \Delta \phi(t)} \mathrm{d} t$

This integral is difficult to evaluate analytically for given $\Delta \phi(t)$, but we can simplify the result for certain situations.

For small $v T$ we can use a Taylor-series approximation for $d_{p_{t}, p_{x}}$ and $d_{p_{t}, p_{y}}$, resulting in $\Delta d(t) \approx d_{a} d_{p_{t}, p_{0}}(t) / r$, where $r=\sqrt{\left(d_{a} / 2\right)^{2}+d_{t x, r x}^{2}}$. The signal power estimate then becomes

$\mathcal{S}_{s}(f) \approx \frac{1}{T} S_{s}(f) \int_{-T / 2}^{T / 2} e^{-j 2 \pi f \frac{d_{a} v t}{c r}} \mathrm{~d} t=S_{s}(f) \frac{\sin \left(\frac{\pi v T f d_{a}}{r c}\right)}{\left(\frac{\pi v T f d_{a}}{r c}\right)}$

To maximally lose $1 \mathrm{~dB}$ of estimated signal power, $v T f d_{a} \lesssim$ $0.36 r c$.

For very large $v T$, the integral is mainly determined by the transmitter being at the far left or the far right of the receiver, so we find $\Delta d(t) \approx \pm d_{a}$, resulting in

$$
\mathcal{S}_{s}(f) \approx S_{s}(f) \cos \left(2 \pi f \frac{d_{a}}{c}\right)
$$

To guarantee a maximum loss of $1 \mathrm{~dB}$ of estimated signal power for arbitrary measurement time, $d_{a} \lesssim 0.1 \frac{c}{f}=0.1 \lambda$.

We can numerically evaluate the integral of (8) for some practical cases. Consider the detection of a wireless microphone, operating at a center frequency of $500 \mathrm{MHz}$, at a distance $d_{t x, r x}=20 \mathrm{~m}$. The two antennas of the XCSA are $10 \mathrm{~cm}$ apart $\left(d_{a} \approx 0.17 \lambda\right)$. With a velocity of $1.6 \mathrm{~m} / \mathrm{s}$ (a person carrying the microphone), the maximum measurement time $T_{\max }$ to lose not more than $1 \mathrm{~dB}$ of signal power is $T_{\max } \approx 43 \mathrm{~s}$, which is much longer than required by IEEE 802.22. Next, consider the detection of a signal in the ISMband at $2.4 \mathrm{GHz}$ with a laptop that has two antennas at a 
distance of $30 \mathrm{~cm}\left(d_{a}=2.4 \lambda\right)$. The signal is transmitted from a car moving at $30 \mathrm{~m} / \mathrm{s}$ with $d_{t x, r x}=50 \mathrm{~m}$. Now we find $T_{\max } \approx 190 \mathrm{~ms}$. Note that using the approximate (9), we find $T_{\max } \approx 250 \mathrm{~ms}$.

These practical cases show that only for large receive antenna distances and fast-moving transmitters and/or excessive measurement time, the signal can be decorrelated significantly. Under these LOS-assumptions, it is clear that a two-antenna XCSA is applicable in many situations.

\section{Fading}

Not all connections are LOS (or have a large specular component), but must rely on a multitude of multi-path reflections. Diversity is often used to improve the performance of a communications system in these fading channels, and is based on the idea that with multiple antennas, the probability that at least one of them is not in a deep fade is increased. The simplest fading channel is a Rayleigh fading channel, where the instantaneous amplitude $A=\sqrt{X^{2}+Y^{2}}$ is Rayleigh distributed, and the phase is uniformly distributed. Here, $X$ and $Y$ are the real and imaginary parts of the received equivalent baseband signal, both normally distributed with zero mean and $\operatorname{var}[X]=\operatorname{var}[Y]=\sigma^{2} / 2$. The output power of an energy detector with one antenna will then be $\mathcal{P}_{s}=\mathbb{E}\left[A^{2}\right]=\sigma^{2}=P_{s}$. We wish to compare the signal power at the output of the two-antenna XCSA with the signal power at the output of the one-antenna XCSA, where the oneantenna XCSA will give the same signal power as any energy detector using one antenna.

The crosscorrelation system is sensitive to phase variations during the measurement, as was established in section IV-B. Therefore, a good channel model is required that describes the phase-correlation over time and location. Because we do not have that at our disposal yet, here we present a simplified model to serve as a first starting point, where we assume a channel in which the amplitudes of the signal undergo (correlated) fading, but the phase difference of the signal between the two antennas remains constant during the measurement. Note that a constant phase difference over frequency between the two antennas of the receiver can be handled by taking the absolute value as in the LOS-connection, so here we assume a phase difference of 0 .

With received signal amplitudes $A_{1}$ and $A_{2}$ at antenna 1 and 2 , respectively, the crosscorrelator will output $\mathcal{P}_{s}=$ $\frac{1}{T} \int_{\langle T\rangle} A_{1}(t) A_{2}(t) \mathrm{d} t$ as power. In the case of fading, $A_{1}(t)$ and $A_{2}(t)$ are random processes, with $A_{i} \geq 0$. We assume that the average signal power received by both antennas is equal $\left(\mathbb{E}\left[A_{1}^{2}\right]=\mathbb{E}\left[A_{2}^{2}\right]\right.$ ), which is a well-accepted model [9]. When the fading is fully correlated, i.e., $A_{1}=A_{2}$, we get $\mathcal{P}_{s}=\sigma^{2}=$ $P_{s}$ as for the single-antenna case. For independent fading, we get $\mathcal{P}_{s}=\mathbb{E}\left[A_{1} A_{2}\right]=\mathbb{E}\left[A_{1}\right] \mathbb{E}\left[A_{2}\right]=\sqrt{\frac{\pi}{4}} \sigma \sqrt{\frac{\pi}{4}} \sigma=\frac{\pi}{4} \sigma^{2}$. These are the two extreme cases, so for correlated fading the result will be somewhere in between: $\frac{\pi}{4} P_{s} \leq \mathcal{P}_{s} \leq P_{s}$. Maximally $10 \log _{10} \frac{4}{\pi} \approx 1 \mathrm{~dB}$ of signal power is lost, provided the relative phase does not change during the measurements.

\section{CONCLUSIONS}

Crosscorrelation spectrum sensing with one antenna, followed by a splitter and two receivers, has been shown to significantly lower the noise level of an energy detector for spectrum sensing. In this work, we proposed to use a separate antenna for each receiver to lower NF and hence measurement time by removing the splitter, as well as to reduce the residual noise correlation. We identified the different mechanisms that still cause residual noise correlation as mutual coupling, spatial correlation, and man-made noise, which will ultimately limit the reduction of the noise level. Presented measurements verify the partial decorrelation of noise using two antennas, but indicates that multipath effects can play a significant role.

We also showed that the use of two antennas can significantly degrade the signal power that is detected. This is caused by decorrelation of the signal, due to 1 ) the physical distance between the antennas, 2) the presence of relative signal phase shift between the antennas due to Doppler-shift, and 3) the presence of fading. Our calculations indicate that in static and LOS-connections the signal power lost is marginal, making two-antenna crosscorrelation spectrum sensing an interesting solution to more reliable energy detection that deserves further exploration.

\section{ACKNOWLEDGMENT}

We would like to thank F. van Vliet and M. Bentum for fruitful discussions, ASTRON for providing the dipole antennas used in the measurements, and $\mathrm{H}$. de Vries and G. Wienk for practical assistance. This research is supported by the Dutch Technology Foundation STW, applied science division of NWO and the Technology Program of the Ministry of Economic Affairs (project 08081).

\section{REFERENCES}

[1] R. Tandra and A. Sahai, "SNR walls for signal detection," IEEE J. Sel. Topics Signal Process., vol. 2, no. 1, pp. 4-17, 2008.

[2] M. S. Oude Alink, E. A. M. Klumperink, M. C. M. Soer, A. B. J. Kokkeler, and B. Nauta, "A 50MHz-to- $1.5 \mathrm{GHz}$ cross-correlation CMOS spectrum analyzer for cognitive radio with $89 \mathrm{~dB}$ SFDR in $1 \mathrm{MHz}$ RBW," in Proc. 4th IEEE Symp. on New Frontiers in Dynamic Spectrum Access Networks (DySPAN), 6-9 Apr. 2010, pp. 1-6.

[3] R. Tandra and A. Sahai, "Noise calibration, delay coherence and SNR walls for signal detection," in Proc. 3rd IEEE Symp. on New Frontiers in Dynamic Spectrum Access Networks (DySPAN), 2008, pp. 1-11.

[4] M. Heskamp and C. Slump, "Sub-noise primary user detection by crosscorrelation," in Proc. Int. Conf. on Communications (ICC), Jun. 2009.

[5] J. H. Shirley and R. W. Fairbridge, Eds., Encyclopedia of Planetary Sciences. Springer, 1997.

[6] C. A. Balanis, Antenna Theory, 3rd ed. Wiley, 2005

[7] C. P. Domizioli, B. L. Hughes, K. G. Gard, and G. Lazzi, "Noise correlation in compact diversity receivers," IEEE Trans. Commun., vol. 58, no. 5, pp. 1426-1436, 2010.

[8] D. Childers and I. Reed, "Space-time cross-correlation functions for antenna array elements in a noise field," IEEE Trans. Inf. Theory, vol. 11, no. 2, pp. 182 - 190, apr 1965.

[9] L. Fang, G. Bi, and A. C. Kot, "New method of performance analysis for diversity reception with correlated rayleigh-fading signals," IEEE Trans. Veh. Technol., vol. 49, no. 5, pp. 1807-1812, 2000. 